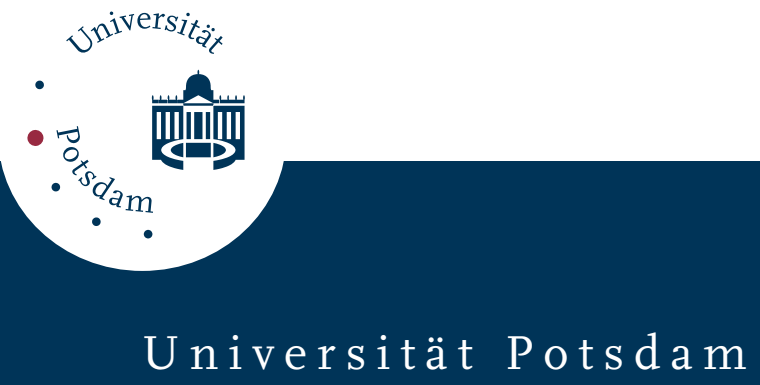

Margret Selting

\title{
Fremdkorrekturen als Manifestationsformen von Verständigungsproblemen
}

first published in:

Zeitschrift für Sprachwissenschaft 6 (1987) 1, ISSN: 0721-9067, S. 37-58

Postprint published at the Institutional Repository of the Potsdam University:

In: Postprints der Universität Potsdam

Philosophische Reihe ; 43

http://opus.kobv.de/ubp/volltexte/2010/4188/

http://nbn-resolving.de/urn:nbn:de:kobv:517-opus-41887

Postprints der Universität Potsdam

Philosophische Reihe ; 43 


\section{Margret Selting}

\section{Fremdkorrekturen als Manifestationsformen von Verständigungsproblemen}

Fremdinitiierte Fremdkorrekturen werden dargestellt als Manifestationen von Formulierungs- oder Verstehensproblemen, die dem Partner zugeschrieben werden. Es werden verschiedene Typen von Fremdkorrekturen analysiert, die sich formal durch ihre syntaktische und/oder prosodische Struktur unterscheiden und für die je typspezifische Gemeinsamkeitsunterstellungen und Problemzuschreibungen rekonstruierbar sind. Bei einigen nicht eindeutig zuordenbaren Fremdkorrekturen lassen der Typ der Fremdkorrektur im Kontext oder die Abfolge von Elementen verschiedener Korrekturtypen darauf schließen, daß die Interaktionspartner die Korrekturtypen untereinander in einer Präferenzstruktur ordnen. Diese Präferenzstruktur unter den Fremdkorrekturen unterscheidet sich jedoch von der an anderer Stelle beschriebenen Präferenzstruktur bei der Manifestation und Bearbeitung sich selbst zugeschriebener Verstehensprobleme durch die weitergehendere Aufrechterhaltung von Gemeinsamkeitsunterstellungen. ${ }^{1}$

\section{Einleitung}

Im Rahmen gesprächsanalytischer Forschung sind seit Mitte der 70er Jahre u.a. sogenannte ,Reparatursequenzen“ ins Zentrum des Interesses gerückt. Die von Schegloff/Jefferson/Sacks 1977 getroffenen Unterscheidungen zwischen Selbstund Fremdreparaturen, und als Spezialfall derselben zwischen Selbst- und Fremdkorrekturen sowie zwischen Selbst- und Fremdinitiierungen von Reparaturen und Korrekturen sind von zahlreichen Autoren aufgegriffen und mittlerweile zum ,Festbestand“ der Gesprächsanalyse geworden (vgl. Levinson 1983: Kap. 6, Streeck 1983, Auer 1984, Kasper 1984, Mazeland 1984, Rehbein 1984, West 1984, Gülich/Kotschi 1985, Henrici/Herlemann 1985 u.a.). Die Analyseperspektive bleibt jedoch bei den meisten dieser Arbeiten auf das Funktionieren der Interaktion als System gerichtet, auf die Aktivitäten der Teilnehmer aus der Perspektive der Aufrechterhaltung oder Reparatur der geordneten Interaktion. Die Perspektive der Teilnehmer, ihre Interpretation dessen, was sie da reparieren, kommt - obwohl gerade die Ethnomethodologie sonst die Teilnehmerbezogenheit von Kategorien stets hervorhebt - nicht in den Blick.

1 Für wichtige Hinweise, die zur Überarbeitung einer ersten Fassung dieses Papiers führten, danke ich insbesondere Franz Januschek (Universität Oldenburg). Weiterhin möchte ich den Mitarbeitern der Bürgerberatung, in der ich das der Analyse zugrundeliegende Korpus erhoben habe, und dem Institut für deutsche Sprache in Mannheim, das mir das ,Sonderkorpus Sozialamtsgespräche“ zur Verfügung stellte, für ihre Kooperationsbereitschaft danken. 
In der vorliegenden Arbeit möchte ich zeigen, daß zumindest einige Fälle von „exponierten fremdinitiierten Fremdkorrekturen“ $-d$.h. Sequenzen, in denen der Interaktionspartner, der die Korrektur in einem eigenen Turn initiiert und auch durchführt, das zu korrigierende Element nicht selbst produziert hat (vgl. Jefferson 1982) - wesentlich differenzierter als in bisherigen Arbeiten beschrieben werden können, wenn man sie auf die Teilnehmerperspektiven bezogen als Manifestationen von dem Partner zugeschriebenen Verständigungsproblemen analysiert, bei denen Zuschreibungen wie ,du hast mich / etwas falsch verstanden / formuliert" rekonstruierbar sind. Ich werde zeigen, daß sich verschiedene Korrekturtypen unterscheiden lassen, die auf unterschiedliche Typen von Verständigungsproblemen verweisen. Typunterscheidend fungieren die syntaktische, semantische und prosodische Struktur der Sequenz und die je rekonstruierbaren Gemeinsamkeitsunterstellungen. (Für eine komplementäre Analyse von fremdinitiierten Selbstreparaturen als Manifestationen von sich selbst zugeschriebenen Verstehensproblemen siehe Selting 1987R.)

Schegloff/Jefferson/Sacks 1977 kamen aufgrund der Analyse ihrer Daten zu dem Ergebnis, daß unter den von ihnen beschriebenen Reparaturtypen Präferenzstrukturen bestehen: Selbstinitiierungen von Reparaturen sind präferierter als Fremdinitiierungen, und Selbstkorrekturen sind präferierter als Fremdkorrekturen. Diese Präferenzstrukturen drücken sich zum einen in der Vorkommenshäufigkeit aus, zum anderen - und vielleicht relevanter - im Sequenzformat, in der Position, an der sie vorkommen: Nach Schegloff et al. lassen Sprecher, wenn sie schon eine Fremdkorrektur vornehmen, häufig zunächst eine Pause entstehen, um dem vorherigen Sprecher die Gelegenheit zu geben, sich selbst zu korrigieren. Oder sie formulieren nur die ,fremdinitiierte Korrekturinitiierung " und überlassen die Durchführung der Korrektur dem vorherigen Sprecher selbst. Desgleichen werden Fremdkorrekturen oft erst nach einer Verständigungssicherungssequenz vorgenommen. Schließlich werden Fremdkorrekturen oft in der Form ,,moduliert" formuliert, d.h., der Sprecher spielt sie durch einen Witz herunter oder ,"tarnt" sie als Nachfrage oder Vergewisserungsfrage (vgl. Schegloff/Jefferson/Sacks 1977: $375 \mathrm{ff}$.).

Darüber hinaus zeigen sich in der vorliegenden Analyse Präferenzstrukturen innerhalb der jeweiligen Reparaturtypen, wonach ,gravierendere" Problemtypen weniger gern zugeschrieben werden als weniger ,gravierende“ Problemtypen. (Zu komplementären Präferenzstrukturen bei sogenanņten fremdinitiierten Selbstreparaturen siehe Selting 1987R und 1987V; zur Analyse von Präferenzstrukturen bei ähnlichen Sequenzen in der Kommunikation zwischen Lehrern und Schülern im schulischen Kontext vgl. Mazeland 1984.)

Beide Typen von Präferenzstrukturen lassen sich plausibel erklären, wenn man anders als Schegloff et al. die Teilnehmerperspektive berücksichtigt: Bei Fremdkorrekturen schreibt ein Sprecher seinem Partner ein Problem zu, das dieser nicht selbst signalisiert hat. Solche Aktivitäten bergen die Gefahr von Imageverletzungen in sich (vgl. Goffman 1978). Je gravierender das zugeschrie- 
bene Problem ist, desto schwerwiegender kann auch die Imageverletzung die weitere Interaktion beeinträchtigen.

Die Daten, auf denen meine Analyse basiert, entstammen einem größeren Korpus von Gesprächen zwischen Klienten und Behördenmitarbeitern in verschiedenen Behörden in Großstädten Nordrhein-Westfalens. In diesem Korpus finden sich zwar viele Sequenzen, in denen ein Interaktionspartner sich selbst ein Verstehensproblem zuschreibt, aber nur ca. 20 Sequenzen der hier analysierten Art, in denen ein Interaktionspartner eine Fremdkorrektur durchführt. Aus dieser kleinen Zahl von Sequenzen und aus dem spezifischen Kontext, dem die Beispiele entstammen, ergibt sich, daß die vorliegende Analyse nur eine eingeschränkte Gültigkeit beanspruchen kann: Sie ist zu verstehen als ein erster Versuch der differenzierteren Analyse von Fremdkorrekturen, von der ich hoffe, daß sie sich an weiteren Analysen bestätigen und gegebenenfalls kontextspezifisch modifizieren läßt.

Im folgenden werde ich zunächst an einem Beispiel das Sequenzformat bzw. das Problembehandlungsschema und die Analysekategorien für die interaktive Behandlung von dem Partner zugeschriebenen Verständigungsproblemen entwickeln. Danach werde ich in Abschnitt 3 die unterschiedlichen Korrekturtypen darstellen und herausarbeiten, daß sie auf je unterschiedliche Problemtypen verweisen. Im Abschnitt 4 werde ich der Frage nachgehen, wie die Korrekturtypen untereinander von den Interaktionspartnern unterschiedlich gewichtet und in einer Präferenzstruktur geordnet sind. Schließlich wird in Abschnitt 5 auf einige Unterschiede zwischen der Behandlung von sich selbst und dem Partner zugeschriebenen Problemen eingegangen, die vor allem in den für die Problemtypen rekonstruierbaren Gemeinsamkeitsunterstellungen liegen.

\section{Das Problembehandlungsschema und die Analysekategorien}

Im folgenden Gesprächsausschnitt (1) formulieren $\mathrm{K}$ und $\mathrm{S}$ nacheinander in den durch Pfeile gekennzeichneten Zeilen jeweils eine ,fremdinitiierte Fremdkorrektur" und konstituieren damit eine Sequenz, die man als zwei aufeinanderfolgende und sich überlappende Korrektursequenzen analysieren kann: ${ }^{2}$

2 Bei der Transkription der Gesprächsausschnitte wurden folgende Transkriptionskonventionen zugrundegelegt:

Intonation und Charakterisierung der Sprechweise:

$\left.\begin{array}{ll}\mathrm{x}_{1} & =\text { fallende Intonation } \\ \mathrm{x}^{1} & =\text { steigende Intonation } \\ \mathrm{x}- & =\text { gleichbleibende Intonation }\end{array}\right\} \begin{aligned} & \text { notiert am Ende einer Einheit, } \\ & \text { Richtung gilt ab letzter akzen- } \\ & \text { aber dáerter Silbe } \\ & \text { sícher }\end{aligned}$


(1) BB7/5B: $56-61$

Korrektursequenzen

56 S: un den- Schwérbehindertqausweis, dện

57 [S: brau ich natürlich áuch, ne'

$\rightarrow 58 \mathrm{~K}: \quad$ Personálausweis,

$\left.\rightarrow \quad \begin{array}{rl}59 \\ 60\end{array}\right] \mathrm{S}:$ neee' den Schwérbehindertnausweis ${ }_{\text {, }}$ den hát

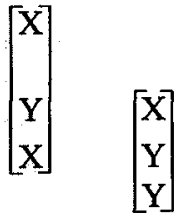

$61 \mathrm{~K}$ : se ja noch ních, das ís beántracht, aber-

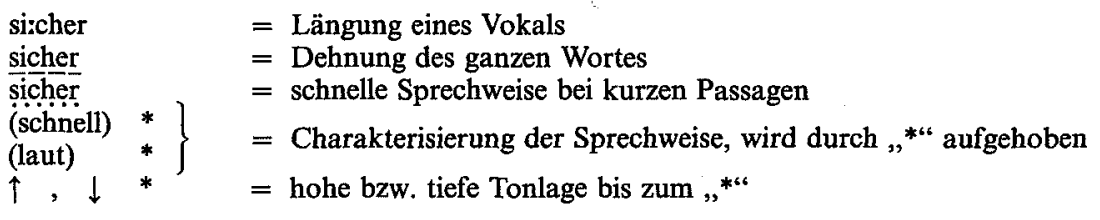

Sprechpausen:

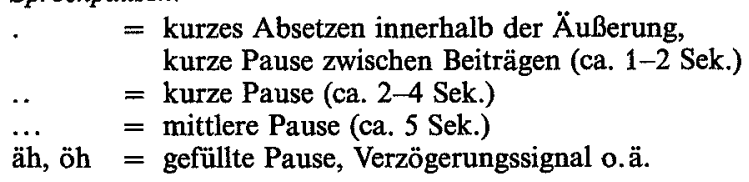

Verständlichkeit:

$\begin{array}{ll}(.),(. .) & =\text { unverständliche Passage, je nach Länge } \\ \text { (? er kommt } & =\text { vermuteter Wortlaut, nicht sicher rekonstruierbar } \\ \text { al(s)so } & =\text { nicht mit Sicherheit identifizierbare Laute }\end{array}$

Rezeptionssignale:

$\mathrm{hm}$

ja

nein

nee

eingipflige Signale, in der Regel mit steigender ('), fallender $\left(\begin{array}{l}() \\ )\end{array}\right.$ oder gleichbleibender $(-)$ Intonation

$\mathrm{mhm}$

jaa neiin $(=[$ nain $])$

zweigipflige Signale, in der Regel mit

fallend-steigender (') oder steigend-

nee $(=[$ ne: $])$

fallender $(1)$ Intonation; Doppelbeschreibung

von Vokalen/Diphthongen bezieht sich nicht auf Sprechweise!

(Dagegen bezeichnet [ne'] in der Regel ein Dialogsignal, mit dem ein Sprecher ein Rezeptionssignal des Rezipienten anfordert.)

Sonstige Konventionen:

$(($ schnieft $))=$ Charakterisierung außersprachlicher Vorgänge/Handlungen

i= Abbruch, Selbstunterbrechung eines Sprechers

Sprecherwechsel:

B: bei Sprecherwechseln wird in die Partiturschreibweise übergegangen, untereinanK: der stehendes in der Klammer ist parallel zu lesen

Zur Notation von phonetischen und morphophonemischen Merkmalen der Sprechstile siehe die Erläuterungen in Selting (1987V).

Ausdrücke aus dem Transkript:

Ausdrücke aus dem Transkript werden durch \lceil\rceil ausgezeichnet. 
In beiden Sequenzen signalisiert die Sprecherin der Fremdkorrektur, die Korrigierende, daß für sie ein bestimmtes Element der vorausgegangenen Äußerung der vorherigen Sprecherin, das somit retrospektiv zum Bezugselement der Korrektur wird, für sie problematisch ist und sie es deswegen durch ein anderes Element ersetzt. Mit dieser Ersetzung wird damit ein Problem manifestiert und zugleich bearbeitet, das die Korrigierende jedoch nicht sich selbst, sondern der vorherigen Sprecherin, der Korrigierten, zuschreibt. Dieser Korrigierten wird durch die Korrektur und die damit implizierte Zuschreibung eines Problems die Rolle der Problemverursachenden zugewiesen: sie hat ein korrekturbedürftiges Element produziert, das nun in einer Nebensequenz behandelt werden soll.

Konstitutiv für eine Problembehandlungs(neben) sequenz ist die auf die Korrektur folgende Reaktion der Korrigierten, da diese signalisiert, ob die Korrigierte die Korrektur als Korrektur bzw. als Problembehandlung ratifiziert oder nicht. Im Gesprächsausschnitt (1) weist in der ersten Korrektursequenz S in Z. 59 die Korrektur der $\mathrm{K}$ aus $\mathrm{Z}$. 58 explizit zurück und wiederholt das Bezugselement; damit wird die Sequenz XYX konstituiert. Wenn man die Wiederholung des Bezugselements durch S in Z. 59 zugleich wiederum als Korrektur der vorausgegangenen Korrektur der $\mathrm{K}$ als Bezugselement interpretiert, dann wird in der zweiten Korrektursequenz diese letzte Korrektur der S von K in Z. 60 mit dem Pronomen [den] wiederaufgenommen und damit bestätigt; hiermit wird die Sequenz XYY konstituiert (vgl. Jefferson 1982).

In beiden Sequenzen geht also hier die Korrigierte explizit auf die Korrektur ein und ratifiziert damit offenbar die Problembehandlung.

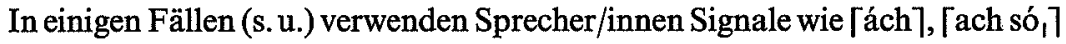
zu Anfang des Ratifizierungsturns und signalisieren damit explizit, daß das ihnen zugeschriebene Problem gelöst ist. Hieraus ergibt sich dann direkte Evidenz, daß der Korrigierte die Korrektur als Problemzuschreibung an ihn als Problemverursachenden interpretiert hat. Meistens wird jedoch die Problemlösung wie in Z. 60 implizit signalisiert, indem das suspendierte übergeordnete Gespräch wiederaufgenommen wird.

Nicht eingegangen bin ich bisher auf die Form der Fremdkorrekturen im Gesprächsausschnitt (1) und auf ihre Beziehungen zu den Bezugselementen. Genau die unterschiedlichen Formen von Fremdkorrekturen sind es jedoch, die eine Einteilung in verschiedene Typen von Fremdkorrekturen zulassen, mit denen dem Problemträger jeweils verschiedene Typen von Verständigungsproblemen zugeschrieben werden. Dies impliziert die Hypothese, daß die Form der Fremdkorrektur eine Problemkategorisierung signalisiert, d.h. eine Zuordnung des zugeschriebenen Problems zu einem bestimmten Problemtyp. Bei der Analyse der Formen und Typen von Fremdkorrekturen spielen die prosodische und syntaktische Struktur der Fremdkorrektur und die semantische Beziehung zwischen Fremdkorrektur und Bezugselement eine typunterscheidende Rolle. Hierauf wird im nächsten Abschnitt eingegangen.

Zuvor noch einige Bemerkungen zur Vorgehensweise, die hier von konversa- 
tionsanalytischen Prinzipien z. T. abweicht. Anders als z. B. bei der Analyse von Manifestationsformen für sich selbst zugeschriebene Verstehensprobleme, wo die Reaktion des Rezipienten der Problemmanifestation eine Rekonstruktion der mit der Problemmanifestation implizierten Problemkategorisierung erlaubt (vgl. Selting 1987V), kann hier die Reaktion des Korrigierten auf die Korrektur nicht für die Typisierung der Korrekturen herangezogen werden. Denn die Korrigierten tragen in der Regel nur noch eine explizite Bestätigung oder Zurückweisung der Korrektur bei, aus der jedoch nicht auf die Problemkategorisierung

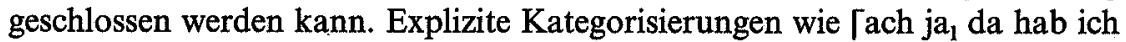
mich versprochen $\left.n_{1}\right]$ kommen in meinen Daten nicht vor.

Die im folgenden unterschiedenen Korrekturtypen ergeben sich zunächst aufgrund der unterschiedlichen Verwendung rein formaler Mittel. Ihre Interpretation als Problemkategorisierung zu bestimmten Problemtypen folgt Plausibilitätserwägungen: Wenn die Analyse der Sequenzen als Problembehandlungssequenzen aufgrund der gelegentlichen Verwendung der Signale 「ach só, $\rceil$ o. ä. nahegelegt wird, dann erscheint auch einleuchtend, daß ein bestimmter Problemtyp zugeschrieben wird. Diese Überlegung wird gestützt durch (a) die systematische Verwendung prosodischer und syntaktischer Strukturen, die mit der semantischen Beziehung zwischen Fremdkorrektur und Bezugselement kookkurrieren, und (b) durch die Rekonstruktion der der Korrektur voraufgegangenen Aktivitäten, die oft eine Interpretation der Korrektur als Zuschreibung eines ganz bestimmten Problemtyps nahelegen.

\section{Korrekturtypen}

Ich unterscheide die folgenden Korrekturtypen:

(1) unmarkierte Ersetzung eines Einzelelements der voraufgegangenen Äußerung,

(2) markierte Ersetzung eines Einzelelements der voraufgegangenen Äußerung,

(3) markierte Ersetzung einer gesamten Bezugsäußerung.

Alle im Korpus vorkommenden Korrekturen werden mit fallender terminaler Intonation realisiert. Als Markierungsformen bei den Korrekturtypen (2) und (3) treten prosodische Markierungen durch die Formulierung auf hoher bzw. höherer globaler Tonlage als in den umliegenden Sequenzen oder durch starke Akzente und/oder äußerungssyntaktische Markierungen durch die Verwendung expliziter Zurückweisungen wie $\lceil$ nee $\rceil$, $\lceil X$ nicht $\rceil$ als Korrektureinleitungen auf. 
3.1. Unmarkierte Ersetzung eines Bezugselements der voraufgegangenen Äußerung

Als Beispiel für diesen Korrekturtyp kann bereits die Korrektur der K in Z. 58 im oben angeführten Gesprächsausschnitt (1) gelten. Es wird ein einzelnes Element der Bezugsäußerung durch ein anderes Element ersetzt: 「Schwérbehin-

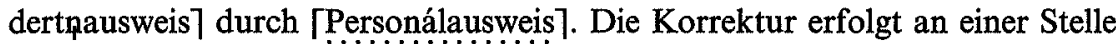
möglicher Redeübergabe, aber bevor $\mathrm{S}$ ihren Turn endgültig abgeschlossen hat. $\mathrm{K}$ formuliert die Korrektur mit schneller Sprechgeschwindigkeit. Sie erscheint wie eine nebenbei und als relativ unauffällig intendierte Aktivität, von der die Korrigierende erwartet, daß die Korrigierte sie akzeptiert. Diese Erwartung läßt sich aufgrund des voraufgegangenen Gesprächs annehmen: $\mathrm{K}$ wollte Informationen über die notwendigen Unterlagen für eine Rundfunkgebührenermäßigung, die sie für eine 「alte Dame † beantragen will. Diese alte Dame war von ihr lediglich als 「alte Dame $\rceil$ mit bestimmten Charakteristika eingeführt worden, nicht jedoch als Schwerbehinderte. $S$, die die 「alte Dame $\rceil$ routinemäßig als Schwerbehinderte eingeordnet hat, thematisiert in Z. 56 zum erstenmal im Gespräch diesen für sie offenbar selbstverständlichen Sachverhalt, indem sie den

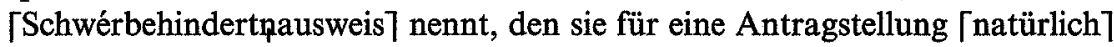
auch brauche. Für $\mathrm{K}$ ist diese Zuordnung aber unerwartet, und sie formuliert ihre Korrektur, offenbar mit der Unterstellung, S habe sich in Z. 56 ,versprochen" und eine falsche Formulierung gewählt.

Ein ähnlicher Fall von unmarkierter Fremdkorrektur liegt im Gesprächsausschnitt (2) vor:

(2) S-31-6: $40-50$

40 B: . wie héißt der Vermieter noch mál' $\mathrm{C}^{\prime}$

41 K:

äh/ ti/ ein a jetzt/

42 K: ich hab das ních bei, (? den ?) letztn- Stréifn, vom/

43 K: wo ich Míete, ich hab ja vorgen Monat Míte bezáhlt,

44 K: nich' . B in- . B/

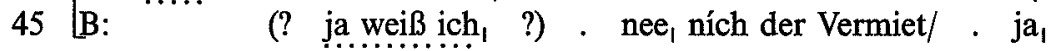

$\rightarrow 46[\mathrm{~K}:$. Lu- a der der Háus- . Sie méin den Háusmeister da,

47 B: ich meine: der Verwálter

48 K: ja (langsamer) Ć heißt der, ${ }^{*}$

49 B: (? wie ?)। $\quad C_{1} \cdot$ äh- $\cdot$ mit

50 B: dém- . verstehn Se sich glaub ich ganz gút $\mathrm{ne}^{\prime}$

Hier formuliert K in Z. 46 mit der Äußerung 「Sie méin den Háusmeister da \

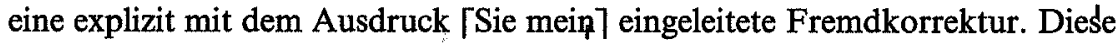
Korrektur erfolgt, nachdem S sich bezüglich der von ihm bezeichneten Person bereits einmal selbst korrigiert hat (Z. 45/47 als Selbstkorrektur in bezug auf 
Z. 40). K glaubt, die bezeichnete Person zu identifizieren, schreibt dem B hier jedoch offenbar das Problem zu, die zutreffende Funktionsbezeichnung 「Háusmeister $\rceil$ im Moment nicht verfügbar zu haben. Dieses Formulierungsproblem hatte B kurz vorher durch die Einleitung der Selbstkorrektur 「nee, ních der Vermiet/ $\rceil$ und durch die Längung des Vokals [ə] in [meine: 7 (Z. 47) selbst signalisiert. In diesem Fall geht $S$ jedoch auf die Korrektur nicht ein und ratifiziert damit die Problembehandlung nicht. Vielmehr setzt er das übergeordnete $\mathrm{Ge}$ spräch sogleich fort mit einer Identifizierungssequenz bezïglich des Namens der in Rede stehenden Person.

Wenn die hier explizierte Interpretation der Gesprächsausschnitte zutrifft, so signalisiert die Korrigierende jeweils mit der unmarkierten Korrektur eines einzelnen Bezugselements dem vorherigen Sprecher, daß sie diesem ein Formulierungsproblem ${ }^{3}$ in bezug auf das ersetzte Element zuschreibt, das sie mit ihrer Korrektur behandelt. Diese Korrektur wird weder prosodisch mit hoher Tonlage und/oder starken Akzenten, noch äußerungssyntaktisch durch ein explizites Zurückweisungssignal ,herausgehoben“", sondern eher durch leise oder schnelle Sprechweise „heruntergespielt" in der Interaktion. Ob hier tatsächlich nur ein Formulierungsproblem zugeschrieben wird oder aber ob etwa im Beispiel (2) zwar ein „Sachverhaltsproblem", nämlich das Nichtkennen der richtigen Personenzuordnung, zugeschrieben, aus Gründen der Imagewahrung 0 . ä. jedoch nur die Zuschreibung eines Formulierungsproblems signalisiert wird, ist nicht ersichtlich. Mit der Zuschreibung eines Formulierungsproblems bleibt die Unterstellung der Sachkompetenz oder der Geteiltheit der zugrundeliegenden Wissensrahmen erhalten; dem Problemverursacher wird nur eine Wortverwechslung, ein momentanes Zuordnungsproblem, zugeschrieben.

\subsection{Markierte Ersetzung eines Einzelelements der voraufgegangenen Äußerung}

In den Gesprächsausschnitten (3) und (4) korrigiert jeweils K in den Z. 98 bzw. 586 den vorherigen Sprecher S bzw. B in markierter Form.

(3) S-34-5: 91-102

$91\left[\mathrm{~K}:\right.$. ách (schnell) sehn $\mathrm{Se}_{1}$ ich wollt Sie wat fragn ${ }^{*}$

92 S: ja

$93 \mathrm{~K}:$ (schnell) ham Síe den (? Áufhebungsbescheid ?) gekri/

$94 \mathrm{~K}$ : vom Ärbeitsamt gekricht* híer (schnell) hingeschickt

3 In dem Sinne, daß aus der Sicht des Korrigierenden der Korrigierte durch seine korrekturbedürftige Formulierung ein Verständigungsproblem verursacht hat, wird hier auch die Zuschreibung eines Formulierungsproblems unter die generelle Analyse von 1 rimdkorrekturen als Fremdzuschreibung eines Verstehens- oder Verständigungsproblems subsumiert. Zum Unterschied bei der Verwendung der Begriffe 'Verstehens-' und 'Formulierungsproblem' hier und bei Kindt/Weingarten 1984 siehe Selting (1987V). 


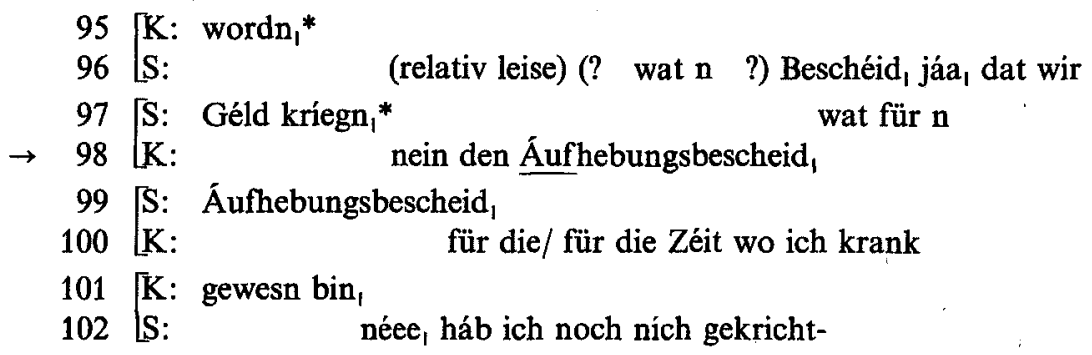

(4) S-31-6: $\quad 583-590$

583 B: . (relativ leise) nee, das brauchn Se auch nich dat $_{1}$

584 B: Ding könn Se só mitnehm, hádder gesacht, äh̆h- hám wa wa

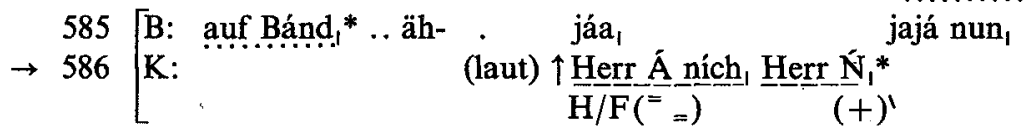

587 B: der A hat dat aber (? ángegebm ?),

588 K: (leise) $\mathrm{mhm}^{\mathrm{i}}$. $\mathrm{muß}$

589 K: er mehr wissen als ich $_{1}^{*}$

590 B: (leise) wir wíssen dat jednfalls, ${ }_{1}^{*}$.

Die Markierung im Sinne der Hervorhebung der Korrektur wird im Gesprächs-

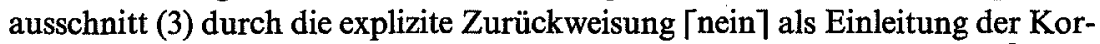
rektur und durch den starken Akzent auf dem ersetzenden Element 「Áufhebungsbescheid $\rceil$ vorgenommen. Im Gesprächsausschnitt (4) findet sich ebenfalls eine explizite Zurückweisung des Bezugselements ( $\left[\right.$ Herr Á ních $\left.\left.{ }_{1}\right\rceil\right)$, des mit dem Pronomen $\lceil e r\rceil$ in $\lceil$ hádder (Z. 584) beibehaltenen Bezugs auf den bereits vorher in Rede stehenden [Herrn A 7 ; zudem wird die gesamte Korrektur mit lauter und gedehnter Sprechweise und auf hoher globaler Tonlage realisiert. Die Akzente

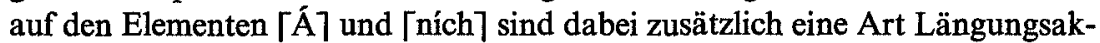
zent, ikonisch darstellbar durch eine Kurve der Art „乙 “", wodurch die Äußerung zusätzlich intonatorisch markiert wird. In beiden Fällen wird auch hier nur ein einzelnes Element der voraufgegangenen Äußerung ersetzt.

Ein weiteres Beispiel für diesen Korrekturtyp ist der Gesprächsausschnitt (5), in dem $\mathrm{K}$ in den Z. 153 und 155-156 beide Markierungsformen jeweils einzeln in aufeinanderfolgenden Fremdkorrekturen verwendet:

\section{(5) S-31-6: 149-157}

149 B: aber der A hat uns ja áuch

150 B: erzählt daß Sie- äh- (jedes Wort einzeln artikuliert)

151 B: in der Wírtschaft Écke Póstweg und-* Erzbergerstraße 
152 B: häufig sind ${ }_{1} \quad$ ja ich wéiß

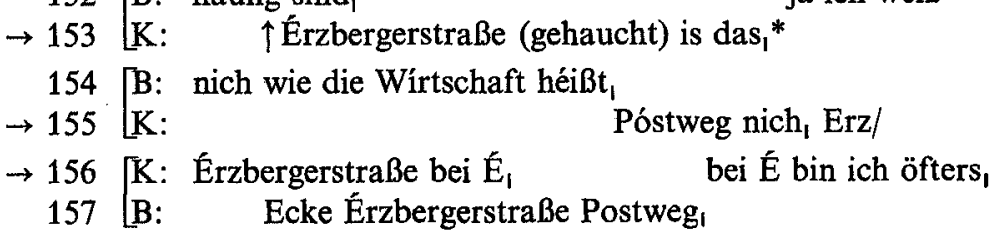

Die Korrektur in Z. 153 ist nur intonatorisch durch die global hohe Tonlage markiert; die Korrektur in Z. 155-156 nur durch die explizite Zurückweisung des Bezugselements.

Insgesamt ergibt sich aus diesen und aus weiteren hier nicht angeführten Beispielen, daß als Markierungsformen entweder eine explizite Zurückweisung des Bezugselements mit nachfolgender prosodisch markierter oder unmarkierter Ersetzung verwendet werden. Oder es wird keine explizite Zurückweisung formuliert, dann aber die Ersetzung immer prosodisch markiert. ${ }^{4}$

In diesen Fällen scheint der Korrigierende dem Korrigierten nicht mehr nur ein Formulierungsproblem zuzuschreiben. Im Beispiel (3) hatte K in Z. 93 wahrscheinlich den Ausdruck [Aufhebungsbescheid] ins Gespräch gebracht. Dieser war in Z. 96-97 von S als ein Ausdruck interpretiert worden, der auf einen Bescheid darüber referiert, daß das Sozialamt Geld bekommt. Diese Zuordnung eines Ausdrucks zu einem Referenten/Sachverhalt wird dann offenbar von K in Z. 98 korrigiert. S nimmt den Ausdruck [Aufhebungsbescheid] in Z.97-99 zwar wieder auf, jedoch in einer eigenen Problemmanifestation, in der er sich selbst ein Bedeutungsverstehensproblem in bezug auf diesen Ausdruck zuschreibt (vgl. Selting 1987V).

Im Gesprächsausschnitt (4) korrigiert $\mathrm{K}$ eine Zuordnung zwischen Person und Sachverhalt: Der Sachverhalt, daß ein Mann gesagt hat, sie brauche bestimmte Möbelstücke nicht zu bezahlen, trifft nicht auf [Herrn A], sondern auf [Herrn N] zu. Im Beispiel (5) schließlich korrigiert K die Zuordnung einer Ortsbezeichnung zu einer Gaststätte, in der sie sich öfters aufhält. Diese Korrektur wird von B in Z. 157 zurückgewiesen.

4 Wenn diese Analyse zutrifft, läßt sich auch für Selbstkorrekturen annehmen, daß die z. B. von Rath 1979 vorgenommene Unterscheidung ,Selbstkorrekturen mit und ohne Korrektursignal“" (Rath 1979: $207 \mathrm{ff}$.) unter alleiniger Berücksichtigung lexikalischer und anderer Signale auf der Textebene unter Vernachlässigung von Prosodie und Intonation, wie auch die von z. B. Levelt/Cutler 1983 vorgenommene Unterscheidung zwischen prosodisch markierten und prosodisch unmarkierten Selbstkorrekturen unter expliziter Nichtberücksichtigung von Verzögerungs- und lexikalischen Korrektursignalen (Levelt/Cutler 1983: 210) jeweils zu kurz greifen. Weiterhin läßt meine Analyse vermuten, daß Gülich/Kotschi 1985 zu kurz greifen, wenn sie Korrektureinleitungssignale als ,Spur“ der Textherstellung auffassen. Damit wird nicht erfaßt, daß diese Korrektursignale offenbar eine typunterscheidende Funktion haben. Ebensowenig wird diese interaktive Funktion erfaßt, wenn man die Korrektureinleitung wie Henrici/Herlemann 1985 als optionale Bestandteile einer Korrektur auffaßt. 
Wenn auch hier der Korrigierende dem Korrigierten ein Problem bei der $\mathrm{Zu}$ ordnung von Sachverhalten zu anderen Sachverhalten, Personen oder Ausdrükken oder kurz ein "Sachverhaltsproblem" zuschreibt, so bleibt dennoch eine Bedeutungszuordenbarkeitsunterstellung aufrechterhalten: Durch die Verwendung definiter Ausdrücke in der Korrektur signalisiert der Korrigierende, daß er unterstellt, der Korrigierte könne diesen Ausdrücken Referenten bzw. Sachverhalte zuordnen (vgl. Searle 1969: 81 ff., Schegloff 1972). Somit wird das zugeschriebene Problem lediglich als Irrtum, nicht aber als fehlendes Wissen zugeschrieben.

Bei beiden bisher dargestellten Korrekturtypen, der unmarkierten und der markierten Ersetzung eines Einzelelements der voraufgegangenen Äußerung, stehen das Bezugselement und die Ersetzung in einer semantischen Beziehung zueinander: Es werden nahe beieinander liegende Ausdrücke oder Sachverhalte innerhalb eines als gemeinsam unterstellten Wissensrahmens gegeneinander gestellt, aber die Fokussierung auf einen damit als gemeinsam unterstellten Sachverhalt wird beibehalten. Es findet keine Refokussierung (vgl. Kallmeyer 1978) statt.

\subsection{Markierte Ersetzung einer gesamten Bezugsäußerung}

Während bei den bisherigen Korrekturtypen ein Einzelelement der voraufgegangenen Äußerung durch ein anderes Element ersetzt wurde, wobei sich aber die gemeinsame Fokussierung nicht verändert bzw. verändern sollte, wird in den folgenden Fällen die gesamte voraufgegangene Äußerung ersetzt durch eine neue Äußerung. Hierbei hat die Korrektur die Form eines syntaktisch vollständigen „Satzes“ mit finitem Verb. Mit der Korrektur wird in der Regel eine Bezugsbehauptung oder -darstellung eines Sachverhalts zurückgewiesen und ersetzt durch eine Gegenbehauptung oder -darstellung. Die Sequenz von Bezugsbehauptung und Gegenbehauptung ähnelt dem Beginn einer Argumentation. Hiermit geht oft eine Refokussierung des Gesprächsgegenstands einher:

(6) S-34-5: 134-150

134 C: die Kránkenkasse hat uns doch schon ángerufn ${ }_{1}$ ? bis viertn

135 S: ja $a_{1}$ wir kríegn doch

136 [C: elftn, ?)

137 S: Kránkengeld kríegn wir,

$\rightarrow 138$ K: (schnell) ja aber ohne Áufhebungsbescheid

$\rightarrow 139[\mathrm{~K}:$ aber kriegn Se* kéin Geld, (schnell) \&óhne

140 S:

(laut) $\uparrow \underline{\text { hé }}^{\text {** }}$

141 K: Aufhebungsbescheid, *

wenn ich dén

142 S:

wíe ohne Aufhebungsbescheid, (? wat denn 
143 [K: nich einge/ den muß ich zum (.)

$\rightarrow 144$ S: für $\mathrm{n}$ ?)Áufhebungsbescheid, vom (laut) Árbeitsamt* krisse

[kbISe]

$\rightarrow 145$ S: kein Geld mehr,

146 K: den Áufhebungsbescheid muß ich habm vonnat

[fonat]

147 K: Arbeitsamt- sons krieg ich vom Arbeit Kránken- k/ äh vom

148 K: Kránken- äh von(ne) Krankenkasse kéin Géld

149 S:

(leise) $\downarrow j a_{1}$ verstéh

150 [S: ich zwar nich' aber-*

Im Beispiel (6) finden sich zwei Fremdkorrekturen, die nur prosodisch markiert sind: In Z. 138-139 korrigiert $\mathrm{K}$ die zuvor von $\mathrm{C}$ und S explizierte Darstellung, sie bekämen Geld von der Krankenkasse, durch die Gegendarstellung, dieses Geld bekäme das Sozialamt aber nicht, ohne daß der [Aufhebungsbescheid $\rceil$ bei der Krankenkasse vorliege. Die Korrektur enthält als prosodische Markierung den starken Akzent auf dem Element [kéin 7, mithin auf dem im Gesprächskontext kontrastiven Element. In der folgenden Zeile 140 manifestiert $\mathrm{S}$ in bezug auf diese Korrektur des K ein lokales Erwartungsproblem (vgl. Selting 1987R), d.h., er signalisiert, daß der in der Korrektur behauptete Sachverhalt mit seinen Erwartungen unvereinbar ist. Nachdem $\mathrm{K}$ seine Darstellung in Z. 139/141 noch einmal mit einem Akzent auf dem für $\mathrm{S}$ unerwarteten Element 「óhne † bestärkt hat, manifestiert dann $S$ in Z. 142/144 ein lokales Bedeutungsverstehensproblem (vgl. ebd.), das von $\mathrm{K}$ zum Beispiel mit einer Explikation des Hintergrundes seiner Behauptung, die in dem folgenden Äußerungsfragment $\lceil$ den muß ich zum (.) $\rceil$ (Z. 143) auch vermutlich begonnen wird, bearbeitet werden kann.

Allerdings wird diese angefangene Problembearbeitung von S mit der Äußerung [vom (laut) Árbeitsamt* krisse kein Geld mehr ${ }_{1}$ ] unterbrochen. Hiermit korrigiert $\mathrm{S}$ die gesamte bisherige Darstellung des $\mathrm{K}$ und bezieht sich dabei offenbar auf einen ganz anderen Wissensrahmen als den, den $\mathrm{K} \mathrm{zu}$ explizieren versucht: nicht die Krankenkasse, sondern das Arbeitsamt wird als Geldquelle genannt, und nicht das Sozialamt, sondern $\mathrm{K}$ selbst wird als Empfänger des Geldes angegeben. Diese Korrektur ist prosodisch durch die laute Sprechweise und den starken Akzent auf [Ärbeitsamt $\rceil$ markiert und bezieht sich auf den gesamten hier thematisierten Wissensrahmen des $\mathrm{K}$, der mit dem Wissensrahmen des $\mathrm{S}$ unvereinbar ist. $\mathrm{K}$ reagiert hierauf mit einer Reformulierung seiner bereits vorher in wesentlichen Teilen formulierten Sachverhaltsdarstellung und weist damit die Korrektur zurück. Die Sequenz wird von S in Z.149-150 mit einer Relevanzrückstufung beendet.

Eine ähnliche Korrektur liegt auch im Beispiel (7) vor, allerdings nur durch eine Relevanzrückstufung als Einleitung markiert: 
(7) BB7/5 B: 41-53

41 [S: un dann könn Sie béides mitnehm $m_{1}$. daß die Dáme

42 K: jaa

$43 \mathrm{~S}:$ das unterschreibt, né . un dánn machen wa s hier

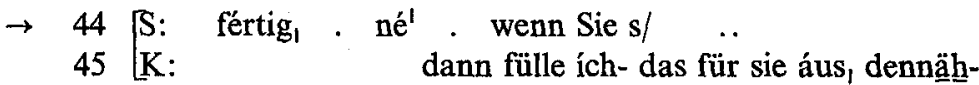

$\rightarrow 46 \mathrm{~S}: \quad$ áusfülln könn wa s auch hier- . wíchtig sind die

$\rightarrow 47$ S: Únterschriftn, . nél

48 K: \&áchäh $h_{1}$ (etwas schneller) wíchti sind

49 K: die Únterschriftn ${ }^{*}$. was bráuchen Se enn noch annäh-

50 S: wichti/ das Áusfülln (...) ।

$51 \mathrm{~K}$ : müssen Sie irgendwelche noch- .

Hier expliziert K in Z. 45, daß sie einen Antrag auf Rundfunkgebührenermäßigung für eine 「alte Dame† ausfüllen will. S stuft in Z. 44/46-47 zunächst die Relevanz dieser Antragsausfüllung herunter und stuft anschließend einen anderen Aspekt, nämlich die Unterschriften, explizit in seiner Relevanz hoch. In der Reaktion der $\mathrm{K}$ in Z. 48-49 signalisiert diese, daß sie diese Relevanzrückstufung und -hochstufung hier als Korrektur interpretiert. Mit [áchäh $\left.{ }_{1}\right]$ signalisiert sie die Lösung des ihr von S zugeschriebenen Verstehensproblems - offenbar eine aufgrund ihrer Erwartungen falsch gesetzte Relevanz - und nimmt dann die Korrektur in einem Ratifizierungsturn auf, bevor sie das suspendierte Gespräch wieder aufnimmt.

Im Gesprächsausschnitt (8) wird eine äußerungssyntaktisch und prosodisch markierte Fremdkorrektur formuliert:

(8) S-33-4: 249-259

249 S: den (..) siehse gár

250 [S: nich' den Háns'

251 K:

(? is der ?)

252 [S: jetz zu- . vórnehm gewordn'

$\rightarrow 253$ K: Stádt

$\rightarrow 254$ K: Montáge da ${ }_{1}^{*} \quad$ nee $_{1}$

255 S: ah so, ich hab gedacht der wär zu vornehm gewordn,

256 K: der is immer auf Montáge, die Fráu die die treff ich

257 S: álles klar,

$258 \mathrm{~K}$ : ốfter inne $\mathrm{Stadt}_{1} \mathrm{ne}^{\mathrm{I}}$

In diesem Beispiel wird die von S in seiner Frage in Z. 250/252 explizierte An-

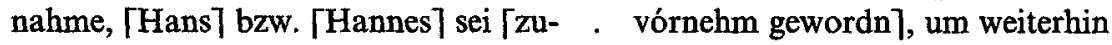
Kontakt zu K zu pflegen, von $\mathrm{K}$ in Z. 253-254 zurückgewiesen und durch die 


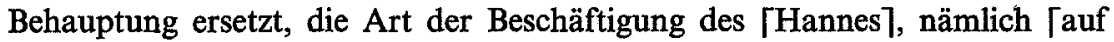
Montáge $\rceil$ zu gehen, sei der Grund für den geringen Kontakt zwischen ihm und K. Hiermit wird eine Inferenz des S aus der von ihm beobachteten Beziehungsveränderung zwischen $\mathrm{K}$ und Hannes von $\mathrm{K}$ zurückgewiesen und korrigiert. Allerdings wurde diese Korrektur hier von $\mathrm{S}$ durch die Formulierung seiner Inferenz als Frage mit steigender Intonation bereits als möglicherweise erwartbar nahegelegt; ${ }^{5}$ interaktiv erhält damit die Korrektur des $\mathrm{K}$ hier einen anderen Status als die bisher diskutierten Korrekturen, die für die Korrigierten zumindest weniger erwartbar erfolgten. Die Korrektur des $\mathrm{K}$ enthält in diesem Fall eine explizite Zurückweisung durch das Element $\lceil$ néee, $\rceil$ sowie eine syntaktisch vollständige Gegenbehauptung, in der $\mathrm{K}$ durch die Partikel 「do (doch) auf die unterstellte Bekanntheit des von ihm behaupteten Sachverhalts verweist. Die gesamte Korrektur wird zudem prosodisch markiert auf hoher globaler Tonlage formuliert. An die Korrektur schließt sich hier ein explizites Problemlösungssignal des S überlappt an (「ah so,, , Z. 255) und eine wechselseitige Versicherung über die offenbar neue Information für S. Die gesamte Problembehandlungssequenz wird von $S$ in $Z .257$ mit dem Signal 「álles klar, 7 beendet.

In den Beispielen (6) bis (8) wird also nicht mehr nur ein Einzelelement der voraufgegangenen Äußerung, sondern ein ganzer Wissensrahmen bzw. eine explizierte Inferenz oder Erwartung durch die Ersetzung der gesamten BezugsäuBerung korrigiert. Die Korrektur unterscheidet sich von den zuvor analysierten Ersetzungen einzelner Bezugselemente durch die Form der syntaktisch vollständigen „Satzstruktur“. Wie bei der markierten Ersetzung eines Einzelelements der voraufgegangenen Äußerung werden als Markierungsformen auch hier die explizite Zurückweisung der Bezugsdarstellung mit nachfolgender prosodisch markierter oder unmarkierter Gegendarstellung und die Gegendarstellung mit obligatorischer prosodischer Markierung verwendet.

Gegenüber den vorher analysierten Korrekturtypen wird bei diesem letzten Korrekturtyp, bei dem der Korrigierende dem Korrigierten also offenbar ein Inferenz- oder Erwartungsproblem zuschreibt, die Unterstellung eines gemeinsamen Wissensrahmens fallen gelassen. Der Korrigierende schreibt dem Korrigierten nicht mehr nur eine Verwechslung oder einen Irrtum zu, sondern ein quasi „falsches Denken“. Deshalb wird eine Explikation des entgegengesetzten Wissensrahmens durch den Korrigierenden notwendig. Allerdings scheint auch hier gelegentlich der Korrigierende, wie z. B. im Gesprächsausschnitt (8), Z. 253,

5 Auer 1984 diskutiert ähnliche Fälle von „Nahelegungen“ für Selbstreparaturen und schlägt daraufhin vor, die von Schegloff/Jefferson/Sacks 1977 entwickelte Typologie von Reparaturen als prototypische Endpunkte eines Kontinuums anzusehen, auf dem es eine Reihe von anderen Zwischen-Techniken gibt, mit denen Interaktionsteilnehmer eine Reparatur nahelegen und aushandeln können. Diese Zwischen-Techniken betreffen, wie Auer zeigt, insbesondere die Initiierung von Reparaturen: zwischen der Selbst- und der Fremdinitiierung steht z. B. die Technik der „Nahelegung“ einer Fremdreparatur. 


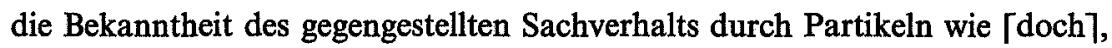

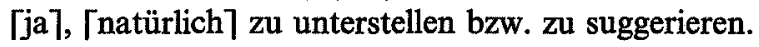

Die hier unterschiedenen Korrekturtypen lassen sich wie in Schema 1 zusammenfassen und den damit zugeschriebenen Verständigungsproblemen zuordnen.

1. Zuschreibung eines Formulierungsproblems

$\left[\begin{array}{l}\text { evtl. Einleitung der Kor- } \\ \text { rektur durch [Sie meip }\end{array}\right]\left[\begin{array}{l}\text { Ersetzung des Bezugselements } \\ (- \text { prosodisch markiert })\end{array}\right]$

2. Zuschreibung eines Irrtums bei der Zuordnung von Sachverhalten $\left[\begin{array}{l}\text { Einleitung der Korrektur } \\ \text { durch Zurückweisung: } \\ \lceil\text { nee }\rceil,\lceil\mathrm{X} \text { nich }\rceil\end{array}\right]$. $\left[\begin{array}{l}\text { Ersetzung des Bezugselements } \\ ( \pm \text { prosodisch markiert })\end{array}\right]$ $\left[\begin{array}{l}\text { Ersetzung des Bezugselements } \\ \text { ( }+ \text { prosodisch markiert })\end{array}\right]$

3. Zuschreibung eines Inferenz- oder Erwartungsproblems

$\left[\begin{array}{l}\begin{array}{l}\text { Einleitung der Korrektur } \\ \text { durch Relevanzrückstufung } \\ \text { oder prosodisch oder stili- } \\ \text { stisch markierte Zurück- } \\ \text { weisung: }\lceil\text { néee, }\rceil, \text { TWeih- } \\ \text { nachtsmann, }\rceil\end{array} \\ {\left[\begin{array}{l}\text { Ersetzung der Bezugsdarstellung } \\ \text { durch Refokussierung/neue Rele- } \\ \text { vanzsetzung oder Gegendarstel- } \\ \text { lung } \\ \text { ( } \pm \text { prosodisch markiert) }\end{array}\right.}\end{array}\right]$

Schema 1: Typen von Fremdkorrekturen und die damit signalisierten Problemzuschreibungen

Die zugeschriebenen Verständigungsprobleme sind z.T. den Typen von Verstehens- und Verständigungsproblemen, die Sprecher bei der Manifestation von sich selbst zugeschriebenen Problemen kategorisieren, sehr ähnlich und verweisen auf unterschiedliche Ebenen des Verstehens und der Verständigung (vgl. Selting 1987V). Es lassen sich dabei die Zuschreibungen von Formulierungsproblemen vergleichen mit der Manifestation von akustischen Verstehensproblemen (Ebene der eher formalen Formulierung und der akustischen Dekodierung einer Äußerung), die Zuschreibungen eines Irrtums bei der Zuordnung von Sachverhalten mit der Manifestation von lokalen Bedeutungsverstehensproblemen (Ebene der Zuordnung von Ausdruck/Sachverhalt zu anderen Sachverhalten) und die Zuschreibung von Inferenz- und Erwartungsproblemen mit der Manifestation lokaler Erwartungsprobleme (Ebene der Erwartungen bzw. der 
Vereinbarkeit mit dem eigenen Wissensrahmen). Wesentliche Unterschiede bestehen jedoch bei den typunterscheidenden Unterstellungen, auf die ich in Abschnitt 5 zurückkomme.

\section{Präferenzstrukturen}

Die hier analysierten Fremdkorrekturen als Fremdzuschreibungen eines Verstehens- oder Verständigungsproblems treten im Gesamtkorpus weniger häufig auf als Manifestationen von sich selbst zugeschriebenen Verstehens- und Verständigungsproblemen. Diese Beobachtung in Verbindung mit den von Schegloff/Jefferson/Sacks 1977 beobachteten unterschiedlichen Sequenzformaten (s. Abschnitt 1) ließe darauf schließen, Fremdkorrekturen als Fremdzuschreibungen von Problemen seien gegenüber der Selbstzuschreibung von Problemen weniger präferiert. Allerdings sind in meinen Daten die von Schegloff et al. beobachteten unterschiedlichen Sequenzformate weniger auffällig; lediglich im Beispiel (4) und in den weiter unten angeführten Beispielen (9) und (10) läßt der Korrigierende eine Pause vor der Fremdkorrektur entstehen; die anderen von den Autoren genannten Phänomene treten in meinem Korpus überhaupt nicht auf. Andere Evidenz für die beschriebene Präferenzstruktur ergibt sich jedoch hier aus Gesprächsausschnitten, in denen beide Manifestationstypen in bezug auf dasselbe Bezugselement vorkommen. Hier zeigt sich, daß eine Reihenfolge, in der die Manifestation eines oder mehrerer sich selbst zugeschriebener Probleme vor der Manifestation eines dem Partner zugeschriebenen Problems in einigen Fällen vorkommt. Die umgekehrte Reihenfolge findet sich dagegen nur im Beispiel (6), wo S in Z. 149-150 mit der Manifestation eines sich selbst zugeschriebenen Problems aber zugleich die Problembehandlung beenden und dieses Problem also nicht bearbeitet haben will.

Die Reihenfolge Manifestation von sich selbst zugeschriebenen Verstehensproblemen vor Fremdkorrekturen findet sich ebenfalls im Beispiel (6) bei $S$ in Z. 140-145 und wurde oben bereits analysiert. Ein weiterer Fall liegt im Gesprächsausschnitt (9) vor:

(9) S-34-3: $250-268$

250 S: nụ̣ páß ma auf Wéihnachtsgeld dat schickn wa Dir zú,

251 [S: né, .. kríss glau(b) ich (? dréi hier fụ̣r ?)

252 K: $\mathrm{ja}_{1}$

für/

253 S: zweihunnert Mark, ne'

254 K:

für. dẹn Jungq áuch was'

(rel. leise) jạ

255 [S: natürlich ${ }^{\prime *}$

$256 \mathrm{~K}:$ ná:i vores Jahr hat er hab ich ja no nix

257 [K: gekricht,

258 S: $\quad$ (rel. leise) $\downarrow$ wíe vorges Jahr hab ich nix 


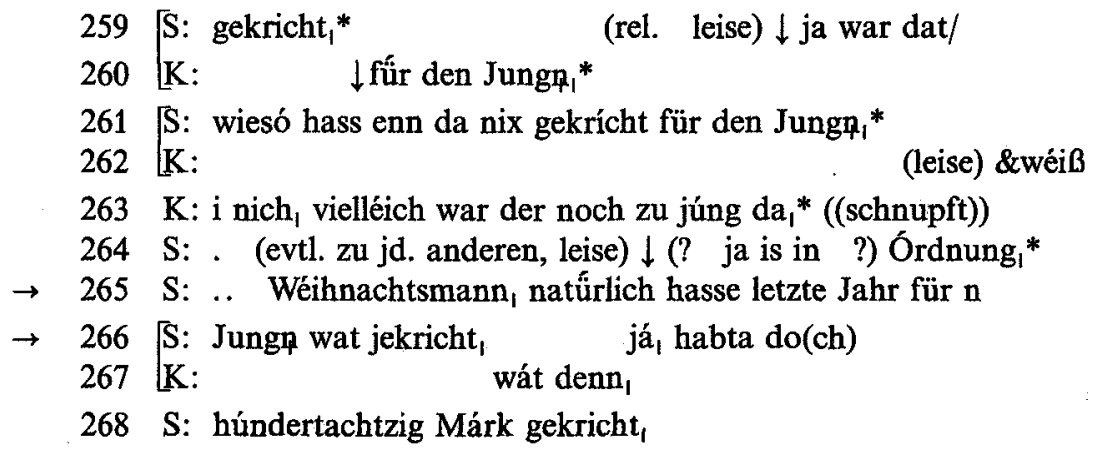

Im weiteren zählt $S$ das gezahlte Weihnachtsgeld für die Familienmitglieder des $\mathrm{K}$ einzeln auf.

Hier manifestiert S in Z. 258--259 zunächst ein sich selbst zugeschriebenes lokales Bedeutungsverstehensproblem, dann in Z. 261 ein sich selbst zugeschriebenes lokales Erwartungsproblem: mit seinen Erwartungen und seinem Wissensrahmen ist unvereinbar, daß $\mathrm{K}$ letztes Jahr für seinen Sohn kein Weihnachtsgeld vom Sozialamt erhalten hat. Als durch die von $\mathrm{K}$ beigetragenen Problembearbeitungen das Problem des S nicht gelöst wird, holt S die Akte des $\mathrm{K}$ zu Hilfe und korrigiert dann erst in Z. 265-266 den K mit einer Fremdkorrektur, die durch die Einleitung mit der auf $\mathrm{K}$ bezogenen Anrede [Wéihnachtsmann $\left.{ }_{1}\right]$ markiert wird. Bevor $\mathrm{S}$ also den $\mathrm{K}$ korrigiert, versichert er sich seines eigenen Verstehens und prüft die Akten, obwohl er vermutlich das dann dem $\mathrm{K}$ zugeschriebene Problem, einen Sachverhalt in seinem Wissensrahmen falsch repräsentiert zu haben, schon früher als Problem vermutete.

Auf eine andere Art von Präferenzstruktur, nämlich eine Präferenzstruktur allein unter den unterschiedlichen Typen von Fremdkorrekturen, weisen vielleicht die Gesprächsausschnitte (10) und (11) hin:

(10) BB3/8 B: 13-23

13 S1: .. a(l)so Stádtplan

14 S1: reicht nich, bíßchen grổßer,

15 K: bißchen grốßer,

16 [S1: es gibt son- son-

17 K: eines zu fümzwanzigtáusend

18 [K: oder $\mathrm{so}_{1}$

$\rightarrow \quad 19$ SS1: $\quad$. (leise) eins zu zwánzigtausend, jál*

20 K: eins zu z/zwánzigtausend hat der,

21 S1: $\quad$ zwánzigtausend

22 S1: is der- . jetz gibt es noch n gró̉ßeren- der hängt-

23 S1: der hängt hier vorne auf dem Flúr, 
Hier formuliert S1 in Z. 19 nach einer kurzen Pause eine unmarkierte Fremdkorrektur, die leise gesprochen und mit dem Zustimmungserwartung signalisierenden Signal [já' $\rceil$ abgeschlossen wird, obwohl er dem K vermutlich nicht nur ein Formulierungs-, sondern auch ein Sachverhaltsproblem zuschreibt. Allerdings hatte $\mathrm{K}$ das Bezugselement der Korrektur durch den Zusatz 「oder so $\rceil$ bereits als ungenau formuliert charakterisiert und damit zwar eine Formulierungskorrektur erwartbar gemacht, eine Sachverhaltskorrektur aber eher verhindert. Da also vor der Korrektur das Bezugselement bereits als ungenau gekennzeichnet ist, kann einerseits als Korrektur des S keine eigentliche Sachverhaltskorrektur mehr erfolgen, ohne das zugeschriebene Problem ,hochzuspielen", sondern nur noch eine Formulierungskorrektur. Wenn dagegen andererseits $S$ dem $K$ auch ein Sachverhaltsproblem, nämlich das Nichtkennen der richtigen Maßstabsbezeichnung, zuschreibt, so läßt seine Behandlung des dem $\mathrm{K}$ zugeschriebenen Problems ebenso wie K's Präventivtechnik auf eine Präferenzstruktur schließen, in der sowohl bei der Selbstzuschreibung wie auch bei der Fremdzuschreibung von Problemen Formulierungsprobleme vor Sachverhaltsproblemen bevorzugt werden.

Auf eine weitere Präferenz von Sachverhaltsproblemen gegenüber Erwartungsproblemen bei Fremdzuschreibungen deutet abschließend der Gesprächsausschnitt (11) hin:

S-34-5: $\quad 116-126$

116 S: (relativ laut) (.) hasse denn hast du denn irgendwie-

117 S: du hast do den Arbeitsún* fähigkeitsbescheinigung

118 [S: gehabt vom Arzt $_{1}$ díe haste zur Kránkenkasse gebracht

119 K:

díe hab

$\rightarrow 120 \mathrm{~K}:$ ich $z u(m) / j a_{1}$ néin, zum Árbeitsamt, diẹ mụ @̣ dạt

$\rightarrow 121$ K: (? Arbeitsamt ?), kricht dat Árbeitsamt ja

122 S: ja, éip kriegt

123 S: Árbeitsamt un éiq Kránkenkasse,

$124 \mathrm{~K}$ :

125 K: schickt der zur Kránkenkasse,

126 S: (relativ leise) ja'*

$127 \mathrm{~K}$ : (schnell) und das Árbeitsamt kricht von mir

In Z. 120 formuliert K nach einem abgebrochenen Äußerungsfragment und dem Signal $\left\lceil\mathrm{ja}_{1}\right\rceil$ die markierte Ersetzung eines Einzelelements der voraufgegangenen Äußerung 「néin, zum Árbeitsamt, ๆ, fügt dann anschließend jedoch die Äußerung [die muß dat (? Arbeitsamt ?), kricht dat Árbeitsamt ja $]$ an. Mit diesem Zusatz wird der Hintergrund seiner Korrektur expliziert; mit der Partikel $\lceil j \mathrm{\rceil}\rceil$ auf die unterstellte Bekanntheit dieses Hintergrundes verwiesen. Es ergeben sich zwei Interpretationsmöglichkeiten hinsichtlich der Funktion dieses Zusatzes: 
(1) Der Zusatz fungiert als Verständigungssicherung für $\mathrm{S}$ in bezug auf die Korrektur.

(2) Mit dem Zusatz, der von der Form her einer markierten Ersetzung einer Bezugsdarstellung gleicht, wird ein dem $\mathrm{S}$ zugeschriebenes, aber nicht als erstes bearbeitetes Erwartungs- bzw. Inferenzproblem bearbeitet.

Beide Interpretationsmöglichkeiten lassen sich vereinbaren, wenn man die erste Korrektur als Manifestation und Bearbeitung eines dem Partner zugeschriebenen Irrtums bei der Zuordnung von Sachverhalten analysiert und den Zusatz als präventive (Mit-)Bearbeitung eines in-Rechnung-gestellten Erwartungsproblems. Diese Interpretation wird gestützt durch die Reaktion des S, der in Z. 122-123 offenbar demonstriert, daß die Information des $K$ für ihn keine neue Information ist: $S$ weist damit die Kategorisierung eines Erwartungsproblems zurück. Im weiteren Gespräch wird jedoch deutlich, daß K's In-Rechnung-Stellung eines Erwartungsproblems bei $\mathrm{S}$ sich durch das Verhalten des $\mathrm{S}$ als ,,berechtigt" bzw. als richtige Hypothese über den Wissensrahmen von S erweist (vgl. insbesondere die Nachfrage $\left[\mathrm{ja}^{1}\right\rceil$ des $S$ in Z. 126). Diese letzte Analyse der Korrekturen bzw. der Korrektur und der Zusatzbearbeitung würde - wenn sie an mehr Daten belegt werden könnte - auf eine Präferenzstruktur hinweisen, wonach die Bearbeitung von Irrtümern als Zuschreibung eines weniger gravierenden Problemtyps bevorzugt würde gegenüber der Zuschreibung eines Erwartungsproblems.

\section{Gemeinsamkeitsunterstellungen bei Selbst- und Fremdzuschreibungen von Verstehens- und Verständigungsproblemen}

Wenn - wie am Schluß von Abschnitt 3.3 ausgeführt - die Typen von Fremdkorrekturen als Fremdzuschreibungen und die in Selting (1987V) und (1987R) beschriebenen Selbstzuschreibungen von Verstehens- und Verständigungsproblemen auf z.T. ähnliche oder dieselben Problemtypen auf denselben Ebenen des Verstehens und der Verständigung bezogen werden können, so unterscheiden sie sich dennoch in den je signalisierten typunterscheidenden Gemeinsamkeitsunterstellungen.

Bei der Behandlung sich selbst zugeschriebener Verstehensprobleme werden mit jedem weniger präferierten Problemtyp grundlegendere Gemeinsamkeitsunterstellungen zurückgewiesen. Aus Platzgründen liste ich die Problemtypen mit ihren typunterscheidenden Unterstellungen hier nur auf (für eine genaue Darstellung siehe Selting (1987V: Kap. 3): 
Problemtyp

(akustisches

Verstehensproblem)

Referenzproblem

lokales Bedeutungs-

verstehensproblem

lokales

Erwartungsproblem typunterscheidende

Unterstellung

[+ Verstehbarkeit $]$

$\left[\begin{array}{l}+ \text { Bedeutungszuordbarkeit } \\ + \text { prinzipielle Reziprozität }\end{array}\right]$

$\left[\begin{array}{l}- \text { Bedeutungszuordbarkeit } \\ + \text { prinzipielle Reziprozität }\end{array}\right]$

[-prinzipielle Reziprozität $]$ präferierterer

Problemtyp

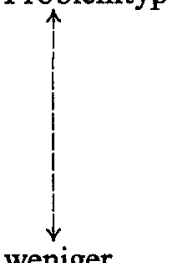

weniger

präferierter

Problemtyp

(Bei den typunterscheidenden Unterstellungen steht das Zeichen „, “ für die Aufrechterhaltung, das Zeichen ,,- “ für das Fallenlassen der betreffenden Unterstellung.)

Im Unterschied zu diesen Problembehandlungen fällt bei den hier analysierten Behandlungen von dem anderen zugeschriebenen Problemen auf, daß durch die Verwendung referierender, meist definiter Ausdrücke bei der unmarkierten und markierten Ersetzung eines Einzelelements der voraufgegangenen Äußerung und durch die gelegentliche Verwendung der Partikeln ja, doch, natürlich u. a. bei der Ersetzung einer Bezugsdarstellung Gemeinsamkeitsunterstellungen weitgehender aufrechterhalten werden. Auch wenn bei der Ersetzung einer Bezugsdarstellung die Unterstellung eines gemeinsamen Wissensrahmens fallengelassen wird, so wird z. T. doch immer noch nahegelegt, der Korrigierte hätte den ersetzenden Sachverhalt nur ,vergessen“, es bestünde aber kein prinzipielles Problem bezüglich der prinzipiellen Reziprozität zwischen beiden. Der Korrigierende scheint demzufolge so lange wie möglich nach der Maxime vorzugehen „Wenn ich den anderen schon korrigiere, dann tue ich wenigstens so, als wüßte er den von mir entgegengesetzten Sachverhalt und hat ihn nur (im Moment) vergessen“.

Hieraus sind prinzipiell gegenläufige Strategien bei Fremdkorrekturen rekonstruierbar: Die Durchführung einer Fremdkorrektur scheint prinzipiell als potentielle Imagebedrohung vom Korrigierten interpretierbar zu sein. Dagegen signalisieren die genannten Beibehaltungen der Gemeinsamkeitsunterstellungen eine Imagewahrung, die die Korrektur quasi in ihrer Wirkung neutralisiert (vgl. Goffman 1978).

Mit dieser Analyse von Fremdkorrekturen aus der Perspektive der Gesprächsteilnehmer und mit der Rekonstruktion der potentiellen Interpretierbarkeit von Fremdkorrekturen als Imageverletzungen werden die von Schegloff/Jefferson/Sacks (1977) und die von mir beobachteten Präferenzstrukturen plausibel erklärbar: Fremdkorrekturen als Fremdzuschreibungen von 
Problemen haben deshalb eine geringere Vorkommenshäufigkeit und ein anderes Sequenzformat als die Manifestationen von sich selbst zugeschriebenen Problemen und Fremdkorrekturen werden deshalb erst nach Verständigungssicherungssequenzen oder der Manifestation eines sich selbst zugeschriebenen Problems vorgenommen, weil sie dem anderen auch je etwas ganz anderes, ,antun“. Sie greifen quasi in die Kommunikationstätigkeit des anderen als autonomen Subjekts ein und tun damit etwas, das dieser andere als gravierende Störung der Interaktionsbeziehung interpretieren könnte. Hiermit ist dann auch plausibel erklärbar, daß die mit den typunterscheidenden syntaktischen und prosodischen Mitteln signalisierten unterschiedlichen Korrekturtypen unterschiedlich gravierende Problemtypen zuschreiben und daß demzufolge der Korrigierende mit der Zuschreibung gravierenderer Problemtypen vorsichtiger ist als mit der Zuschreibung weniger gravierender Probleme.

\section{Literaturnachweis}

[Auer 1984] Auer, J.C.P.: Referential problems in conversation. - In: Journal of Pragmatics 8 (1984), 627-648.

[Goffman 1978] Goffman, Erving: Interaktionsrituale. - Frankfurt/M.: Suhrkamp 1978.

[Gülich/Kotschi 1985] Gülich, Elisabeth/Kotschi Thomas: Reformulierungshandlungen als Mittel der Textkonstitution. Untersuchungen zu französischen Texten aus mündlicher Kommunikation. - In: Satz, Text, sprachliche Handlung. Hrsg. von Wolfgang Motsch. Berlin: Akademie-Verlag. = Studia grammatica. 25. [Erscheint demnächst].

[Henrici/Herlemann 1985] Henrici, Gert / Herlemann, Brigitte: Mündliche Korrekturen im Fremdsprachenunterricht. - Universität Bielefeld 1985. [Manuskript].

[Jefferson 1982] Jefferson, Gail: On exposed and embedded correction in conversation. - In: Studium Linguistik 14 (1982), 58-68.

[Kallmeyer 1978] Kallmeyer, Werner: Fokuswechsel und Fokussierungen als Aktivitäten der Gesprächskonstitution. - In: Sprechen - Handeln - Interaktion. Hrsg. von R. MeyerHermann. - Tübingen: Niemeyer 1978. S. 191-241.

[Kasper 1984] Kasper, Gabriele: Repair in foreign language teaching. - In: Studies in Second Language Acquisition 7 (1984), 200-215.

[Kindt/Weingarten 1984] Kindt, Walther / Weingarten, Rüdiger: Verständigungsprobleme. - In: Deutsche Sprache 3 (1984), 193-218.

[Levelt/Cutler 1983] Levelt, Willem J.M. / Cutler, Anne: Prosodic marking in speech repair. - In: Journal of Semantics 2,2 (1983), 205-217.

[Levinson 1983] Levinson, Stephen C.: Pragmatics. - Cambridge: Cambridge University Press 1983.

[Mazeland 1984]. Mazeland, Harrie: „Was kostet denn iks?”“ - semantisch-pragmatische Analyse einer Frage. - Seminar für allgemeine Sprachwissenschaft, Universität Düsseldorf, Dezember 1984. = Arbeitspapier $11 \mathrm{im}$ DFG-Projekt „Analysemethoden von Unterrichtskommunikation".

[Rath 1979] Rath, Rainer: Kommunikationspraxis. - Göttingen: Vandenhoeck und Ruprecht 1979.

[Rehbein 1984] Rehbein, Jochen: Reparative Handlungsmuster und ihre Verwendung im Fremdsprachenunterricht. - ROLIG-papir 30/84, Roskilde universitetsCenter 1984.

[Schegloff 1972] Schegloff, Emanuel A.: Notes on a conversational practice: formulating 
place. - In: Language and Social Context. Ed. by P.P. Giglioli. - Harmondsworth: Penguin 1972. S. 95-135.

[Schegloff / Jefferson / Sacks 1977] Schegloff, Emanuel A. / Jefferson, Gail / Sacks, Harvey: The preference for self-correction in the organization of repair in conversation. - In: Language 53 (1977), 361-382.

[Searle 1969] Searle, John R.: Speech acts. An essay in the philosophy of language. London: Cambridge University Press 1969.

[Selting 1987R] Selting, Margret: Reparaturen und lokale Verstehensprobleme. Oder: Zur Binnenstruktur von Reparatursequenzen. - [Manuskript. Erscheint in: Linguistische Berichte 108 (1987)].

[Selting 1987V]-: Verständigungsprobleme. Eine empirische Analyse am Bëispiel der Bürger-Verwaltungs-Kommunikation. [Erscheint: Tübingen: Niemeyer 1987].

[Streeck 1983] Streeck, Jürgen: Konversationsanalyse. Ein Reparaturversuch. - In: Zeitschrift für Sprachwissenschaft 2,1 (1983), 72-104

[West 1984] West, Candace: Medical misfires: mishearings, misgivings, and misunderstandings in physician-patient dialogues. - In: Discourse Processes 7 (1984), 107-134. 